\title{
A Survey of Cognitive Radio Access to TV White Spaces
}

\author{
Maziar Nekovee ${ }^{1,2}$ \\ ${ }^{1}$ BT Innovate and Design, Polaris 134, Adastral Park, Martlesham, Suffolk IP5 3RE, UK \\ ${ }^{2}$ Centre for Computational Science, University College London, 20 Gordon Street, London WC1H OAJ, UK
}

Correspondence should be addressed to Maziar Nekovee, maziar.nekovee@bt.com

Received 2 November 2009; Accepted 6 April 2010

Academic Editor: Fred Daneshgaran

Copyright ( 2010 Maziar Nekovee. This is an open access article distributed under the Creative Commons Attribution License, which permits unrestricted use, distribution, and reproduction in any medium, provided the original work is properly cited.

\begin{abstract}
Cognitive radio is being intensively researched as the enabling technology for license-exempt access to the so-called TV White Spaces (TVWS), large portions of spectrum in the UHF/VHF bands which become available on a geographical basis after digital switchover. Both in the US, and more recently, in the UK the regulators have given conditional endorsement to this new mode of access. This paper reviews the state-of-the-art in technology, regulation, and standardisation of cognitive access to TVWS. It examines the spectrum opportunity and commercial use cases associated with this form of secondary access.
\end{abstract}

\section{Introduction}

A cognitive radio [1] consists of a cognitive engine (CE), which contains algorithms and toolboxes for radio environment sensing, machine-learning, and reasoning and decision making, and a configurable radio platform, which could be a Software Defined Radio (SDR), that basically does what it is told by the CE. The concept of Cognitive Radio (CR) was first described by Mitola and Maguire [2] as "transforming radio nodes from blind executors of predefined protocols to radio-domain-aware intelligent agents that search out ways to deliver the services that the user wants even if that user does not know how to obtain them". The ideal CR knows everything about the user requirements, the capability of the radio device, the network requirements and the external environment (including the radio environment). It will plan ahead and negotiate for the best part of the spectrum to operate in and at the best power, modulation scheme, and so forth, and manage these resources in real time to satisfy the service and user demands. The ideal CR is currently at the early proof-of-concept stage research, with most of the work taking place in universities.

A much more developed form of the CR technology is cognitive radio for dynamic spectrum access (DSA) [3]. The aim here is to achieve device-centric interference control and dynamic reuse of radio spectrum based on the frequency agility and intelligence offered by cognitive radio technology.
This form of CR technology is currently being intensely researched. However, there is also already significant industry effort towards prototyping, standardisation and commercialisation of the technology. Important industry players with active R\&D efforts in cognitive radio technology include Alcatel-Lucent, Ericsson and Motorola from the mobile equipment industry, BT and Orange from network operators, Philips and Samsung from the consumer electronics industry, HP and Dell from the computer industry, and Microsoft and Google from the Internet/software industry. Dynamic spectrum access may take place in several ways: between a licensed primary system and a license-exempt secondary system, for example, secondary spectrum access to digital TV or military spectrum, within the same primary system, for example, micro-macro sharing of licensed spectrum in 3G/LTE femtocells, and finally among two primary systems, for example, real-time leasing and trading of spectrum between two cellular operators.

The first form of dynamic spectrum access is arguably the most disruptive application of the CR technology, as it enables license-exempt users (end-user devices and base stations) to act as spectrum scavengers. They can identify unused portions of licensed spectrum (also called spectrum holes or White Spaces) and make opportunistic use of this spectrum for their connectivity at times and/or locations where they are not used. Allowing the operation of such scavengers promises to greatly increase the efficiency of 
spectrum usage by preventing exclusively licensed spectrum from being wasted due to low spatial or temporal usage. Mainly for this reason licensed-exempt cognitive access to certain licensed bands is being keenly promoted by the US regulator, the (Federal Communication Commission) FCC [4-7], and more recently also by Ofcom [8-10]. The rationale is to maximise the usage of licensed spectrum through secondary access by cognitive radios and, at the same time, promote rapid introduction of new wireless technologies and services without the need for setting aside any new spectrum for this purpose. Most mobile operators see this from of cognitive access as highly disruptive to their current business model.

In the longer term (3-5 years), we expect that dynamic spectrum access based on cognitive radio will go far beyond opportunistic spectrum access only. As a result of the current trends in spectrum liberalisation, including the availability of licensed spectrum for real-time trading, cognitive devices may be able to access a portfolio of different types of spectrum for their connectivity. This "spectrum portfolio" may include several different type of spectrum: licensed spectrum (e.g., in cellular bands), licensed-exempt spectrum (in the ISM bands), as well as spectrum, that is, acquired in real-time, either through leasing or on a secondary basis. Devices with cognitive functionality will be able to dynamically change their operating spectrum within this portfolio, accessing the best available spectrum on a "justin-time" basis. This may happen either upon instruction from a base station or autonomously by devices themselves. Depending on the user and network requirements devices may pool together and use several spectrum fragments and vacate some or all of them when they are no longer required or when other more suitable ones become available. These requirements may depend on context, application and location and can include price, Quality of Service (QoS), and energy saving.

To date both in the UK [10] and US [5-7] regulators have committed to licence-exempt cognitive access to the so-called TV White Spaces (TVWS). The TVWS spectrum comprises large portions of the UHF/VHF spectrum that become available on a geographical basis for cognitive access as a result of the switchover from analogue to digital TV. The total capacity associated with TVWS is significant. According to modelling studies commissioned by Ofcom over $50 \%$ of locations in the UK are likely to have more that $150 \mathrm{MHz}$ of interleaved spectrum and that even at $90 \%$ of locations around $100 \mathrm{MHz}$ of interleaved spectrum might be available for cognitive access [10]. In addition to TVWS, the defence spectrum may provide another significant capacity opportunity for license-exempt cognitive access. For example, around 30\% of spectrum below $15 \mathrm{GHz}$ is allocated to Defence in the UK. The UK (Ministry of Defence) MoD had until the late 1990's access to spectrum at no or a low cost. However, following the Cave Audit, the Government has committed to releasing a "significant proportion" of the MOD's spectrum between 2008 and 2010. Results form a 2008 study by PA consulting (commissioned jointly by MoD and Ofcom) suggest that [11] there is significant scope for license-exempt use of the released spectrum using cognitive radio technology, both on a spatial and a temporal basis. For example, low power cognitive devices could potentially share with radar if the radar sweep can be detected and the transmission of the cognitive device can be timed to avoid interference.

This paper aims to review the state-of-the-art in technology, regulation, and standardisation of cognitive radio access to TVWS. It also examines the spectrum opportunity, potential business applications, and some of the open research challenges associated with this new form of access, drawing lessons and conclusions from recent recent findings in the UK $[12,13]$, US $[14,15]$, and elsewhere. The rest of this paper is organised as follows. Section 2 provides a brief overview of cognitive radio access to TV White Spaces. In Section 3 the regulatory status and standardisation efforts are reviewed and some of the outstanding research and technology challenges are discussed. In Section 4 we discuss recent results on quantifying the availability of TVWS spectrum for cognitive access in the UK and the US, and describe some of the prominent candidate use cases of this spectrum. We conclude this paper in Section 5.

\section{Cognitive Access to TV White Spaces}

2.1. What are TV White Spaces? Broadcast television services operate in licensed channels in the VHF and UHF portions of the radio spectrum. The regulatory rules in most countries prohibit the use of unlicensed devices in TV bands, with the exception of remote control, medical telemetry devices, and wireless microphones. In most developed countries regulators are currently in the process of requiring TV stations to convert from analogue to digital transmission. This Digital Switchover (DSO) was completed in the US in June 2009, and is expected to be completed in the UK by 2012. A similar switchover process is also underway or being planned (or is already completed) in the rest of the EU and many other countries around the world. After Digital Switchover a portion of TV analogue channels become entirely vacant due to the higher spectrum efficiency of digital TV (DTV). These cleared channels will then be reallocated by regulators to other services through auctions.

In addition to cleared spectrum, after the DTV transition there will be typically a number of TV channels in a given geographic area that are not being used by DTV stations, because such stations would not be able to operate without causing interference to cochannel or adjacent channel stations. However, a transmitter operating on such a locally vacant TV channel at a much lower power level would not need a great (physical) separation from cochannel and adjacent channel TV stations to avoid causing interference. Low power devices can therefore operate on vacant channels in locations that could not be used by TV stations due to interference planning. These vacant TV channels are known as TV White Spaces or Interleaved Spectrum in the language of the UK regulator.

2.2. Detection and Incumbent Protection. Secondary operation of cognitive radios in TV bands relies on the ability of cognitive devices to successfully detect TVWS, and is 
conditioned by regulators on the ability of these devices to avoid harmful interference to licensed users of these bands, which in addition to DTV include also wireless microphones. Both the FCC and Ofcom have considered three methods for ensuring that cognitive devices do not cause harmful interference to incumbent: beacons, geolocation combined with access to a database, and sensing. Currently, the database approach seems to offers the best short-term solution for incumbent detection and interference avoidance. Both in the US and UK regulatory and industry efforts is, therefore, underway to further develop the concepts, algorithms and regulatory framework necessary for this approach.

2.2.1. Beacons. With the beacon method, unlicensed devices only transmit if they receive a control signal (beacon) identifying vacant channels within their service areas. The signal can be received from a TV station, FM broadcast station, or TV band fixed unlicensed transmitter. Without reception of this control signal, no transmissions are permitted. One issue with the control signal method is that it requires a beacon infrastructure to be in place, which needs to be maintained and operated, either by the incumbent or a third party. Furthermore, beacon signals can be lost due to mechanisms similar to the hidden node problem described below.

2.2.2. Geolocation Combined with Database. In this method, a device determines its location and accesses a database to determine the TV channels that are vacant at that location. There are at least three issues associated with this method. There is a need for a new (commercial) entity to build and maintain the database. Devices need to know their location with a prescribed accuracy. For outdoor applications GPS can be used to support these requirements, but in the case of indoor application there are issues with the penetration of GPS deep. Finally, devices need additional connectivity in a different band in order to be able to access the database prior to any transmission in DTV bands. inside buildings.

We note that the latter problems can be addressed in master-slave communication architectures where a master device, for example, an access point or base station, has access to location information and is connected via a wireless or fix link to the Internet. The master node uses its location information to query the geolocation database about TVWS channel availability and based on this information instructs a set of slave devices on the frequencies they can use.

2.2.3. Sensing. Finally, in the sensing method, unlicensed devices autonomously detect the presence of TV signals and only use the channels that are not used by TV broadcaster. Detection of the TV signal can be subject to the hidden node problem, which is depicted in Figure 1. This problem can arise when there is blockage between the unlicensed device and a TV station, but no blockage between the TV station and a TV receiver antenna and no blockage between the unlicensed device and the same TV receiver antenna. In such a case, a cognitive radio may not detect the presence of the TV signal and could start using an occupied channel, causing harmful interference to the TV receiver.

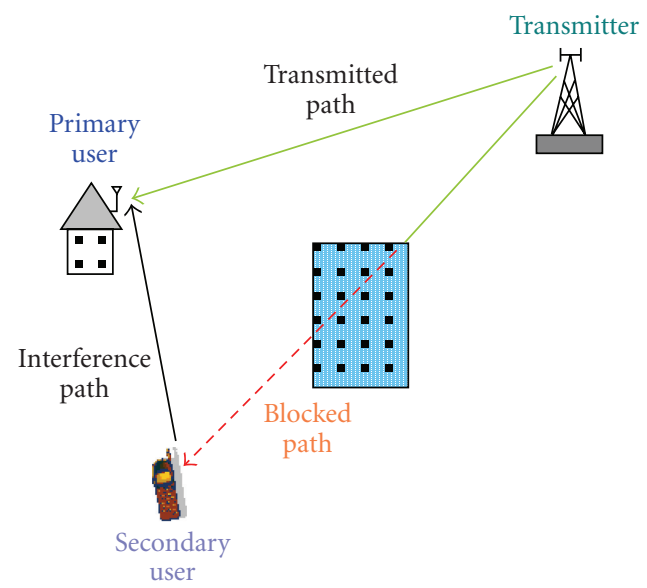

FIGURE 1: The hidden node problem of a sensing-based cognitive radio.

\subsection{Regulatory Developments}

2.3.1. US. In the US the FCC proposed to allow opportunistic access to TV bands already in 2004 [4]. Prototype cognitive radios operating in this mode were put forward to FCC by Adaptrum, (Institute for Infocomm Research) I2R, Microsoft, Motorola, and Philips in 2008. After extensive tests the FCC adopted in November 2008 a Second Report and Order that establishes rules to allow the operation of cognitive devices in TVWS on a license-exempt basis [5-7]. In summary these rules require cognitive devices to use both spectrum sensing and geolocation. In order to minimise the chance of harmful interference due to the hidden node problem FCC has required that cognitive devices should be able to sense both television signals and wireless microphones down to $-114 \mathrm{dBm}$. They must also locate their position to within 50 metres and then consult a database that will inform them about available spectrum in that location [5-7].

Mobile devices may transmit in a locally vacant TV channel at up to $100 \mathrm{~mW}$ unless they are using a channel adjacent to terrestrial television, in which case their transmission power can only be $40 \mathrm{~mW}$. Fixed devices (base stations or customer premises) may transmit at a locally vacant channel at up to $4 \mathrm{~W}$ (EIRP). Devices without geolocation capabilities are also allowed if they are transmitting to a device that has determined its location. In this case, one device would be acting as a master for a network and the other slave devices would operate broadly under its control in terms of the spectrum they would use. Devices that use sensing alone are allowed in principle; however, they must be submitted in advance to the FCC for laboratory and field testing so the FCC can determine whether they are likely to cause harmful interference. The exact process that the FCC will use to determine this has not been specified.

Importantly, the FCC report includes a detailed discussion about whether cognitive access should be licensed, licence-exempt or subject to light licensing. It concludes that the best way to facilitate innovative new applications is via licence-exemption and that licensing would not be 
practicable for many of the new applications envisaged. It also notes that any licenses would be difficult to define and subject to change (e.g., if television coverage was replanned), so the rights awarded would be rather tenuous.

2.3.2. UK. In its Digital Dividend Review Statement released in December 2007 the UK regulator, Ofcom, proposed to "allow licence exempt use of interleaved spectrum for cognitive devices" [8]. Furthermore Ofcom stated that "We see significant scope for cognitive equipments using interleaved spectrum to emerge and to benefit from international economics of scale [8]". In a consultation published on 16 February 2009 [9] Ofcom proposed a number of technical parameters for licence-exempt cognitive use of interleaved spectrum which closely follow those suggested by FCC.

Subsequently, in a statement published on July 12009 Ofcom proposed to allow sensing alone as well as geolocation for incumbent detection [10]. However, it concludes that in the short term the most important mechanism for spectrum detection will be geolocation. Ofcom is suggesting that further work, possibly leading to a consultation specifically on geolocation, is appropriate. Finally Ofcom states that it "will work with stakeholders to further develop the concepts and algorithms necessary for geolocation and expect to consult further on Geo-location later in 2009" [10]. Following this consultation Ofcom published a discussion paper on geolocation for cognitive access in Novemeber 2009.

2.3.3. Worldwide. Work on a pan-European specification for cognitive devices is currently taking place within the SE43 working group of (the European Conference of Postal and Telecommunications Administrations) CEPT. An important aim of this group is to define technical and operational requirements for the operation of cognitive radio systems in TV White Spaces in order to ensure the protection of incumbent services/systems and to investigate the amount of spectrum across Europe that is potentially available as White Spaces. Furthermore on a worldwide scale, agenda item 1.19 of the (World Radiocommunications Conference, 2011) WRC-11 will be considering regulatory measures and their relevance, in order to enable the introduction of software defined radio and cognitive radio systems, based on the results of ongoing ITU-R studies.

2.4. Standardisation and Industry Effort. Industry led research and development on cognitive radio technology has been so far mainly focused in the USA, and is largely driven by the desire of important new players, including Google and Microsoft, to get access to the TVWS spectrum. However, a number of EU-backed and industry-led collaborative projects are currently underway that aim at bringing cognitive radio technology in Europe closer to commercial exploitation. Two major standardisation efforts, which are currently at an advanced stage, are discussed below. It is worth mentioning that in addition to these a number of new standardisation initiatives are underway, which include the IEEE 802.19 and the IEEE 802.11af standards.
2.4.1. The Cognitive Networking Alliance (CogNeA) Standard. The Cognitive Networking Alliance (CogNea) [16] is an open industry association. The Alliance intends to commercialise low power personal/portable Cognitive Radio platforms by enabling and promoting the rapid adoption, regulation, standardisation and multivendor compliance and interoperability of CRs world wide. Alliance board members include ETRI, HP, Philips, Samsung ElectroMechanics, Texas Instruments, and more recently BT. The initial geographical focus area is North America. The initial focus radio spectrum is TV White Spaces.

The Alliance intends to promote TVWS spectrum regulations worldwide, and to establish a recognisable CogNeA brand that indicates a device is CogNeA-compliant and can therefore interoperate with other CogNeA-certified devices from various manufacturers. The Alliance also develops specifications for the Common Cognitive Radio Platform (CCRP) which supports multiple applications [17].

The Alliance intends to bring the standard to an international status, in collaboration with an existing Standards Definition Organisation (SDO), to make it globally accepted. The primary target applications for the CogNeA standard are

(i) in-home high definition multimedia networking and distribution solutions that overcome the whole home coverage problems inherent to solutions using ISM bands,

(ii) unlicensed broadband wireless access for communities/neighbourhoods/campuses.

The standard is developing a Common Radio Platform consisting of the Physical Layer (PHY) and the Media Access Layer (MAC). The PHY consists of the Radio Front End, the Baseband, and the Cognitive Entity, which contains a geo-location block, a sensing block and an Internet access and interference map resources. The MAC carries the Communication/Networking protocol, Air access rules, and interface for the higher layers, such as network and application layers.

ECMA International is currently developing a highspeed wireless networking standard for use in the Television White Spaces, based on the contribution from CogNeA. The standard will employ cognitive radio sensing and database technologies to avoid interference with licensed services and other incumbent users in compliance with the FCC regulatory rules. The first draft of the ECMA/CogNeA PHY and MAC standard for operation in TV White Spaces was published in December 2009 [18, 19].

2.4.2. The IEEE 802.22 Standards. The IEEE 802.22 Working Group [20] has defined an air interface (PHY and MAC) standard based on cognitive radio techniques. The 802.22 standard is being developed for Wireless Regional Area Networks (WRANs). The primary target application of the standard is licensed-exempt broadband wireless access to rural areas in TVWS. The initial geographical focus area is North America. The 802.22 system specifies a fixed point-tomultipoint wireless air interface whereby a base station (BS) manages its own cell and all associated Consumer Premise 
Equipments (CPEs). The network architecture including MAC and PHY are derived from IEEE 802.16 WiMAX. The 802.22 PHY layer is designed to support a system which uses vacant TVWS channels to provide wireless communication access over distances of up to $100 \mathrm{Km}$. The PHY specification is based on Orthogonal Frequency Division Multiple Access (OFDMA) for both the upstream and downstream access.

The IEEE 802.22 standard supports incumbent detection through spectrum sensing (the database approach is optional). The standard specifies inputs and outputs for the sensing function, as well as the performance requirements for the sensing algorithms implemented (e.g., probability of detection, incumbent detection threshold and probability of false alarms). These include energy detector, and cyclostationary and pilot sensing detectors for ATSC DTV signals, and an FFT-based algorithm for detection of wireless microphone signals [21].

The IEEE 802.22 defines a connection oriented and centralised MAC layer. Two important capabilities are introduced in the 802.22 MAC layer to support reliable incumbent detection based on sensing: network-wide quite periods scheduled by each BS during which all transmissions are suspended in order to allow reliable sensing, and channel measurement management to coordinate distributed channel measurement/incumbent detection by CPEs and their reporting to $\mathrm{BS}$.

After an initial accelerated phase, the development of the standard seems to have slowed down during the last year. According to the IEEE 802.22 sources, the standard is currently at the Ballot stage. However, the final completion date for standard is not known yet, and there have been no vendor companies so far to build equipment based on the IEEE 802.22.

\section{Research Challenges}

3.1. High-Precision Spectrum Sensing. In order to minimise the chance of harmful interference due to the hidden node problem both the FCC and Ofcom require that cognitive devices should be able to sense TV signals at detection margins much lower than that of TV receivers $(114 \mathrm{dBm}$ for $6 \mathrm{MHz}$ US channels and $-120 \mathrm{dBm}$ for $8 \mathrm{MHz}$ UK channels) [5-7, 10]. Such weak signals are well below thermal noise, and in the presence of noise uncertainty cannot be detected using the energy detection algorithms that are implemented in the current generation of wireless devices [22]. Recent research, however, shows that such sensing levels may be achieved using more sophisticated sensing algorithms that rely on certain features of incumbent signals which are absent in the noise [23].

In the following we briefly discuss some of these "nonblind" sensing techniques. We note that, unlike energy detection, these algorithms are generally not applicable to sensing signals from wireless microphones, most of which use analogue frequency modulation (FM), and refer the reader to $[24,25]$ for recently proposed algorithms for detection of wireless microphones.

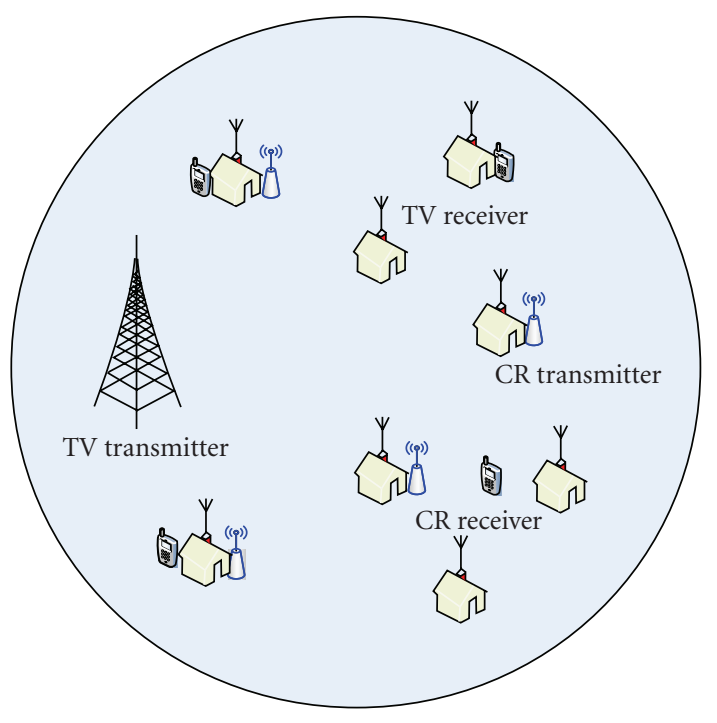

FIGURE 2: In future commercial applications the aggregate power levels of cognitive devices need to be controlled in order to avoid interference to primary receivers.

(i) Pilot detection. These sensing algorithms are specific to ATSC signals, which have a DC pilot at a lower band-edge in a known location relative to the signal. Detection is achieved by setting a threshold either on the amplitude or the location of the pilot signal. Detection based on the location of pilot is in particular robust against noise uncertainty, since the position of the pilot can be pinpointed with high accuracy, even if the amplitude is low due to fading [21].

(ii) Cyclostationary feature detection. Both the ATSC and DVB-T signals are cyclostationary, that is, the means and correlation sequences of these signals exhibit periodicity. Cyclostationary feature detectors were introduced as a complex two-dimensional signal processing technique for recognition of modulated signals in the presence of noise and interference. Recently they have been proposed by a number of authors $[26,27]$ for the detection of weak TV signals in the context of spectrum sensing for cognitive radio.

(iii) Cyclic prefix and autocorrelation detection. OFDM signals, including DVB-T signals, contain a special sequence called cyclic prefix (CP), where the last D bits of the OFDM symbol is copied to the beginning of the symbol. Cyclic prefix detection is similar to energy detection. However, the test statistics used in the algorithm is the energy contained in the cyclic prefix of each OFDM symbol, instead of the whole symbol [28]. Furthermore, due to the presence of CP the autocorrelation function of DVB-T signals show distinct peaks at nonzero values whose amplitude and position could be used to detect the signal from the noise. 
One issue with most of the above sensing algorithms is that they require considerable processing power which may be either not available or not desirable (due to power consumption) in handheld devices. A second problem with high-precision sensing is that the ability of cognitive devices to sense extremely weak TV signals may eliminate the hidden node problem (false negatives) but at the same time it can lead to a situation where a cognitive radio detects TV signals from transmitters that are perhaps hundreds of kilometres away (false positive), thereby removing a considerable portion of usable White Spaces. Very recent studies in the US, for example, indicate that a threshold of $-114 \mathrm{dBm}$ reduces the recoverable White Spaces by a factor of 3 [14]. Even worse, initial modelling studies performed at BT [29] show that in some UK locations, a cognitive device with a $-114 \mathrm{dBm}$ sensitivity level will identify all DTT channels as occupied, and therefore will have no White Space available for its operation if it relies on naive sensing only!

There has been considerable recent research in cooperative detection algorithms, where sensing measurements performed by multiple devices are combined (using either a soft or a hard decision combining method) in order to achieve higher sensing thresholds than is possible by single devices or to deal with the hidden node problem [3032]. Interestingly, cooperative sensing was also considered in Ofcom's consultation on cognitive access as a possible approach to the detection problem [9].

One problem of cooperative sensing is that the achievable detection level depends on several factors, including the number of cooperating cognitive devices and their spatial arrangement $[30,31]$. Therefore, in general it would be difficult to test and certify the detection capability of such cooperating cognitive devices on an individual basis to check device compliance with regulatory requirements [29]. Furthermore this method requires additional communication overhead since local measurements will be collected at system level in order to make a decision, which is then broadcast to all cognitive radios involved.

Due to the above issues we believe that the most promising application of cooperative sensing will be in master-slave communication scenarios, where a computationally powerful master device (e.g., a WiFi access point or a cellular base station) centrally coordinates and process sensing activities of a set of slave devices in combination and uses the result to refine and geographically extend the results of its own sophisticated sensing algorithms.

\subsection{Agile Transmissions and Spectrum Pooling Techniques.} Physical layer transmission techniques that are able to effectively deal with the fragmented nature of TVWS spectrum are a very important component of future cognitive radios. In particular, these techniques must be sufficiently agile to enable unlicensed users to transmit in (locally) available TVWS bands while not interfering with the incumbent users operating at adjacent bands. Moreover, to support throughput-intensive applications, these techniques should be able to achieve high data rates by pooling several (not necessarily contiguous TVWS channels). One technique that seems to meet both these requirements is a variant of orthogonal frequency division multiplexing (OFDM) called non-contiguous OFDM (NC-OFDM) [33, 34]. NCOFDM is capable of deactivating subcarriers across its transmission bandwidth that could potentially interfere with the transmission of other users. Moreover, NC-OFDM can support a high aggregate data rate with the remaining subcarriers, and simultaneously maintain an acceptable level of error robustness. In addition to NC-OFDM several other techniques have been proposed to enable agile waveforming over fragmented spectrum. One prominent example is the use of filterbank multicarrier techniques for such cognitive radio applications [35].

3.3. Multiple Antenna Technologies for Cognitive Radio. The use of antenna diversity or MIMO antenna architecture can provide a significant increase in the spectral efficiency of wireless systems [36, 37]. However, the use of multiple antennas in cognitive radio networks is underdeveloped. One of the major objectives for cognitive radio is to improve the spectrum utilisation. With the advantages offered by MIMO systems, it is therefore logical to exploit potentials in applying the MIMO antenna architecture to cognitive radio networks. Introducing multiple antenna technologies for cognitive radio (CR) may extend the dimension of $\mathrm{CR}$ from the current frequency band and time slot regime even further into spatial domain. A cognitive system using MIMO can significantly improve receiver sensitivity and coverage, hence it may also have impact on the key device parameters such as sensitivity and transmit power in CR, that is, required by regulators.

One issue with the use of MIMO in the context of cognitive access to TVWS is that the typical wavelengths in the UHF bands vary between $0.3-0.6 \mathrm{~m}$. Optimal use of multiple antennas on a single cognitive device, therefore may not be feasible in most applications due to the small footprints involved. However, fixed BWA(Broadband Wireless Access) applications similar to that considered in the IEEE 802.22 that involve large base stations and customer premises may greatly benefit from multiple antenna technologies.

3.4. System-Level Issues. Almost all research so far has focused on a single cognitive device accessing TVWS spectrum. However, the provision of commercial services based on cognitive radio technologies, for example, mobile broadband or wireless home networks, will inevitably involve situations involving multiple cognitive equipments that may belong to either the same or different service providers. Some open research challenges associated with such service scenarios include the following:

(i) estimation and control of aggregate interference from multiple cognitive devices [38] towards primary users,

(ii) politeness (etiquette) rules that achieve fair and efficient sharing of secondary spectrum among competing cognitive radios [9], 


\begin{tabular}{|c|c|c|c|c|c|c|c|c|c|c|c|c|}
\hline \multirow{6}{*}{$\begin{array}{c}\text { Channel } \\
\text { frequency } \\
(\mathrm{MHz})\end{array}$} & 21 & 22 & 23 & 24 & 25 & 26 & 27 & 28 & 29 & 30 & 31 & 32 \\
\hline & $470-478$ & $478-488$ & $488-494$ & 494-502 & $502-510$ & 510-518 & 518-528 & 528-534 & $534-542$ & $542-550$ & $550-558$ & $558-568$ \\
\hline & 33 & 34 & 35 & 36 & 37 & 38 & 39 & 40 & 41 & 42 & 43 & 44 \\
\hline & $568-574$ & 574-582 & $582-600$ & $500-508$ & $508-606$ & 606-614 & $614-622$ & $622-630$ & $630-638$ & $638-648$ & $648-654$ & $654-662$ \\
\hline & 45 & 46 & 47 & 48 & 49 & 50 & 51 & 52 & 53 & 54 & 55 & 56 \\
\hline & $662-670$ & 670-678 & 678-680 & 680-694 & 694-702 & 702-710 & $710-718$ & 718-726 & $726-734$ & $734-742$ & $742-750$ & $750-758$ \\
\hline
\end{tabular}

\begin{tabular}{|c|c|c|c|c|c|c|c|c|c|c|c|}
\hline 57 & 58 & 59 & 60 & 61 & 62 & 63 & 64 & 65 & 66 & 67 & 68 \\
\hline $758-766$ & $766-774$ & $774-782$ & $782-790$ & $790-798$ & $798-806$ & $806-814$ & $814-822$ & $822-830$ & $830-838$ & $838-846$ & $846-854$ \\
\hline
\end{tabular}

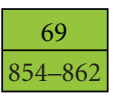

\begin{tabular}{l}
$\square$ Retained/interleaved spectrum \\
Cleared spectrum \\
\hline$\square$ PMSE
\end{tabular}

FIgURe 3: The UK UHF TV bands after completion of Digital Switchover [9].

(iii) spectrum sensing under aggregate interference conditions,

(iv) quantitative understanding of the scalability of secondary spectrum access networks operating in TVWS spectrum.

\section{TVWS Spectrum Availability and Use Cases}

4.1. How Much White Spaces Is Available? Figure 3 shows allocation of the UHF spectrum in the UK after the completion of DSO [10]. The $128 \mathrm{MHz}$ of spectrum marked in green (16 bands) is the cleared spectrum which Ofcom plans to license through auctions. The $256 \mathrm{MHz}$ (32 channels) marked in purple is the interleaved spectrum which can be used on a geographical basis for license-exempt access by using cognitive radio technology. Finally the channel marked in pink is licensed by Ofcom for exclusive access for wireless microphones, and so forth, (PMSE).

From the above chart it appears that there is significant capacity available for cognitive access in the UHF bands. However, due to its secondary nature the availability and frequency decomposition of the UHF spectrum for cognitive access is not the same at all locations and depends also on the power levels used by cognitive devices $[12,14]$. This is an important feature of license-exempt cognitive access to TV bands which distinguish it from, for example, WiFi access to the ISM bands.

Potential commercial applications of TVWS devices will strongly depend on how the availability of this spectrum varies; both from location to location and as a function of transmit power of cognitive devices. A number of recent studies have investigated various aspect of TVWS spectrum in the US $[14,15]$. In the UK we have developed a set of modelling tools that have enabled us to quantify the availability of TVWS spectrum for cognitive access and its variation with location and transmit power.

The first set of these modelling tools [12] makes use of the publicly available maps of DTV coverage in the UK [39] which were generated via computer simulations from the Ofcoms database of location, transmit power, antenna height and transmit frequency of UKs DTV transmitters, and were further validated and improved through direct observations at different locations. It combines these coverage maps with simplified propagation modelling calculations to obtain upper bounds for the vacant TVWS frequencies at any given location as well as a lower-bound estimate for the variations of TVWS spectrum with the transmit power of a cognitive devices.

The computer model for obtaining the upper bounds works as follows [12]. We use the UK National Grid (NG) coordinate system in order to specify the geographical position of any location on the UK map. Given the NG coordinates of a UK location the computer code then maps this location onto the closest grid point on the DTV coverage maps. For a given DTV transmitter this grid point is then evaluated to determine if it falls within the coverage area of that transmitter. If this is the case, then the frequencies associated with the transmitter are tagged as occupied at those locations, otherwise they are tagged as vacant. Repeating this procedure for coverage maps of all DTV transmitters, we then obtain a list of vacant TV frequencies at a given location that can be used by a low-power cognitive devices which is positioned in that location.

In the case of high power cognitive equipments, for example, those considered within the 802.22 standard, the required computations are very intensive. In order to reduce this computational effort, we approximate the actual DTV 
coverage areas by circular disks which were constructed such that each of them entirely encompassed the coverage area of the associated transmitter while also having the minimum possible surface area. With this simplification, it is then computationally straightforward to calculate from the vacant TV frequencies as a function of both position and transmit power of cognitive devices.

A second modelling tool is currently in its final development stage [40]. The tool makes use of Ofcom's published database of DTV transmitter together with highly accurate terrain data and standard UHF radio propagation models to generate contour maps of the received power for every DTV transmitters in the UK. Combining these contour maps with propagation modelling of cognitive devices, the available TVWS for cognitive access at any given location and for any arbitrary power level is then computed with a spatial resolution of $100 \mathrm{~m}$. The terrain data used in this modelling tool is based on the STRM v2 terrain elevation data. The STRM data set resulted from a collaboration effort between NASA and the US National Geographic Intelligence Agency, as well as the participation of the German and the Italian space agencies, and is at present the most complete highresolution digital topographic database of Earth.

We have used our first set of modelling tools to investigate the variations in TVWS as a function of the location and transmit power of cognitive radios, and to examine how constraints on adjacent channel emissions of cognitive radios may affects the results. This analysis provides a realistic view on the potential spectrum opportunity associated with cognitive radio access to TVWS in the UK, and also presents the first quantitative study of the availability and frequency composition of TVWS outside the United States. Figure 4 summarises in a bar-chart the availability of TVWS channels for 18 major population centres in England, Wales and Scotland. The total number of channels available at each location is shown as a green bar. These results show that there are considerable variations in the number of TVWS channels as we move from one UK location to another. For any given location, however, a minimum of 12 channels $(96 \mathrm{MHz})$ is accessible to low-power cognitive devices, while the averaged per location capacity is just over $150 \mathrm{MHz}$.

When a high power cognitive device operates in a vacant TV channel, energy leakage to adjacent channels may cause interference to adjacent frequencies, which may be occupied. Ofcom had raised concerns that operation of low-power cognitive devices on a given channel may also cause adjacentchannel interference for mobile TV receivers that are in close vicinity. Consequently, even in some future use cases, cognitive devices may be constrained not to use vacant channels whose immediate adjacent frequencies are used for mobile TV. The total number of available TVWS after imposing the above adjacent channel constraint are shown as red bars in Figure 4 . It can be seen that imposing the constraint greatly reduces the amount of accessible spectrum in most locations considered (on average the available capacity drops to just below $40 \mathrm{MHz}$ /location).

Recent studies on quantifying the availability of TVWS in the United States were reported in $[14,15]$, and the results are in line with our findings for the UK. In particular a detailed

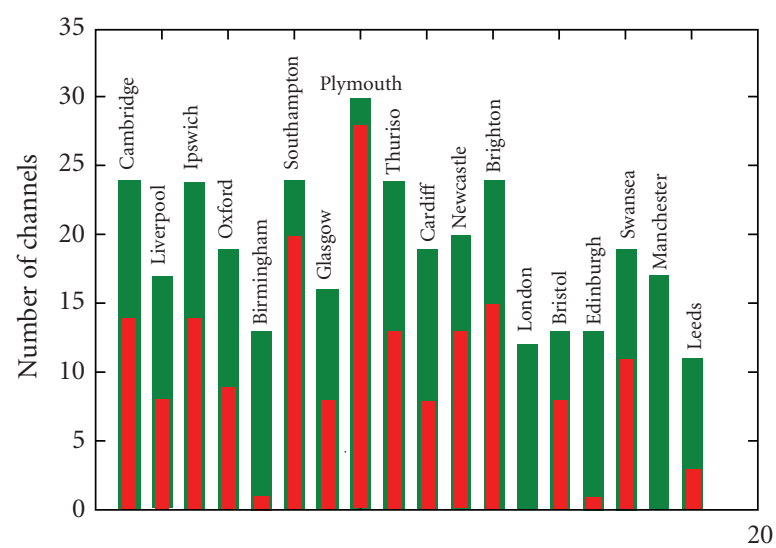

UK location

Adjacent channels permitted

Adjacent channels prohibited

FIGURe 4: Available TVWS capacity for low-power cognitive access in 18 UK locations as obtained from coverage modelling. Results are shown both without (green bars) and with (red bars) considering adjacent channel interference constraint.

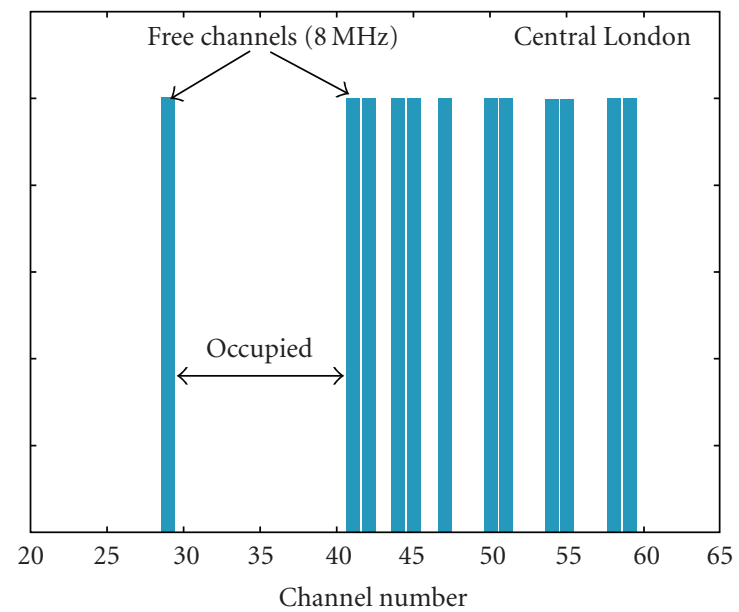

FIgure 5: TVWS channels available for cognitive access in Central London.

study performed in [14] shows that in the US the main channels of relevance are the lower UHF channels where $\sim 15(90 \mathrm{MHz})$ channels per location/per person are available for low power cognitive access. However this number drops significantly (to $\sim 5$ ) when adjacent channels also have to be protected.

In addition to estimating total available TVWS, it is of importance to investigate channel composition of this spectrum. In Figure 5 we show, as an example, channel composition of TVWS in Central London. In this figure vacant channels are shown as blue bars while occupied channels are left black. As can be seen from the figure, the available TVWS channels can be highly non-contiguous. This feature may greatly restrict access to TVWS by most 
current wireless technologies, as modulation schemes implemented in these technologies often require a contiguous portion of the spectrum. In the case of London although a total of $96 \mathrm{MHz}$ spectrum is in principle available, only $16 \mathrm{MHz}$ can be utilised for contiguous frequency access.

We note that the above results were obtained using a combination of highly realistic DTV coverage maps with a simplified pathloss propagation model, which is adequate when considering low-power cognitive devices. Although our results are in good agreement with other independently obtained modelling results, for example, by Ofcom, we are currently not aware of any actual measurements of TVWS avilability in the UK that could be used to directly validate our modelling results. Furthermore, in order to improve the accuracy of our TVWS estimates for high-power cognitive devices, such as those required in rural broadband applications, we are incorporating standard UHF propagation models such as the ITU-R P.1546-3 (LongleyRice) model [41] into our calculations.

4.2. Use Cases. In addition to considerable capacity it offers, which is evident from the discussion in the previous section, an important reason why TVWS spectrum has attracted much interest is an exceptionally attractive combination of bandwidth and coverage. Signals in the VHF/UHF TV bands travel much further than both the WiFi and $3 \mathrm{G}$ signals and penetrate buildings more readily. This in turn means that these bands can be used for a very wide range of potential new services. In addition to broadband wireless access to undeserved areas, other technologically important applications of TVWS spectrum include the following:

(i) wireless distribution networks for future digital homes and smart energy grids,

(ii) licensed-exempt mobile broadband,

(iii) last mile wireless broadband in urban environments,

(iv) cognitive femtocells/cellular communications in TVWS.

In the following paragraphs we will focus our attention on two future use cases of cognitive access to TVWS, which have recently attracted much attention from both industry and research community.

4.2.1. Future Wireless Home Networks. Fuelled by the quick progress of wireless technologies, broadband adoption, and without the burden of spectrum licences, home wireless networking has become in the last few years a pervasive technology. Between 2004 and 2006, home network adoption boomed across Europe, with growth rates surpassing Asia and North America. France and the UK both trebled the number of households with a home network, putting them slightly ahead of the US Italy and Germany still lagged behind but posted notable growth nonetheless. More than 54\% of European households have a computer and a total of $34 \%$ are using WiFi routers. The future wireless home will consist not only of PC, laptops and PDAS wirelessly connected to the Internet but also media servers (High Definition TV, video and audio), access points, computer electronics like wireless cameras and game consoles. Due to sharp decrease in wireless solutions prices and minimum restrictions for access to ISM bands, other domestic applications as well as gas, electricity and water meters will in the future come equipped with radio receivers allowing control, monitoring and easy configuration (the so-called smart grids).

Most of these devices and services support wireless connectivity using one or a number of short-range wireless technologies, such as WiFi (IEEE 802.11), Zigbee (IEEE 802.15.4), and so forth, all operating without the need for a licence in the already congested ISM bands. Home networks of the future operating exclusively in these bands are expected to suffer severe capacity limitations resulting from interference caused by the high device density and limited spectrum availability in the ISM bands. Furthermore, the aggregate interference resulting from these devices is bound to create a high interference burden on the WiFibased provision of broadband wireless access in homes.

Additional capacity offered by secondary access to TVWS has the potential to solve this capacity limitation problem thereby contributing to increasing takeup of wireless home networking and services, and spurring future technological innovation and revenue generation. In particular, some of the most bandwidth-intensive home networks applications (such as multimedia streaming) can be offloaded to TVWS bands hence freeing up the ISM bands for other consumer applications. Our recent system-wide simulation studies show that [13] due to lower operation frequencies home access points operating in the TVWS UHF frequencies can achieve throughput levels that are either higher or comparable to WiFi access points while using significantly lower transmit power levels (two order of magnitudes in $\mathrm{mW}$ ) [13]. An additional benefit here is that a significant saving in energy consumption can be achieved in home networking scenarios by switching from the ISM bands to TVWS bands [13]. Furthermore, according to several recent studies [12, 42] the number of available channels for indoor white space transmissions appear to be very significant.

Protection of incumbents in such home networking scenarios can be achieved using a master-slave architecture where functionalities of spectrum detection and/or geolocation and database access and spectrum assignment are all integrated into the home access point [43]. The access point monitors the availability of spectrum in the ISM and TV bands and instruct customer devices which spectrum to use based on their bandwidth and QoS requirements.

4.2.2. Cognitive/TVWS Femtocells. A femtocell is a small base station of 3G/LTE, or WiMAX technology, controlled by the mobile operator and placed inside the home /small office of the customer. Femtocells are useful when a user experiences bad indoor coverage or its application is too capacity-demanding for indoor conditions. The user may be already inside or going inside a building. Femtocells help maintaining a mobile broadband session or to allow it where it previously was not possible. Current generation femtocells use the same frequencies as mobile networks, hence creating 


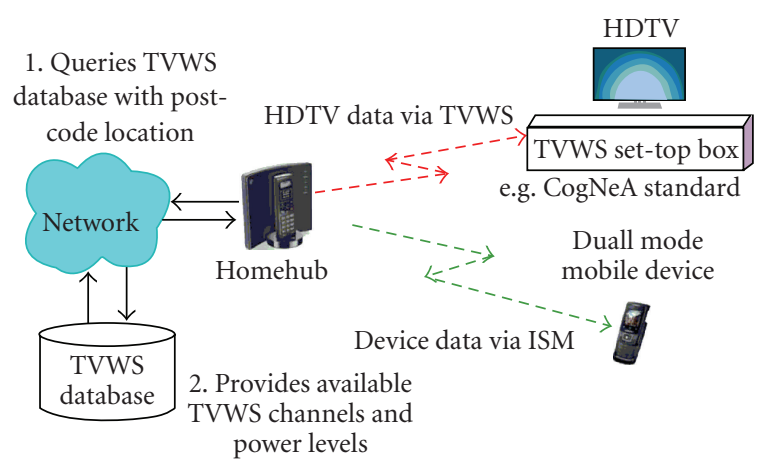

FIgUre 6: Architecture for HDTV distribution using TV White Space spectrum.

a potential source of interference that can be difficult to control since the user femtocell is not controlled by the operator.

Femtocells operating in TVWS would be an alternative to femtocells proprietary technologies that are appearing on the market for dedicated 3G/LTE networks [44]. The main advantage of CR based femtocells compared to traditional femtocells will be reduced or better controlled interference into the operators' network. Another case of great interest to operators is to use CR to backhauling of femtocells (either traditional or CR-based themselves). This allows a mobile operator gain control into the home of the user, should it be outside the DSL coverage or whether the user has another operator delivering broadband to his/her home network.

\section{Conclusions}

In this paper we surveyed the state-of-the-art in cognitive radio access to TV White Spaces. We showed that a regulatory framework for secondary utilisation of TVWS spectrum is well underway both in the US and UK and important steps in this direction are also being taken within the EU and worldwide. Using result from recent quantitative studies of the TVWS availability in the UK and US we illustrated that cognitive access to these bands provides a very significant spectrum opportunity for a range of indoor and outdoor applications and services. In addition to rural broadband, which is the main focus of the IEEE 802.22 standard, these include wireless home networks, mobile broadband, and TVWS femtocells.

However, effective exploitation of this spectrum for such commercial services requires addressing an array of important technology challenges. One of these, high-precision spectrum sensing, has been the subject of numerous research papers while others, including multiple secondary access, aggregate interference control, and agile modulation techniques, have not yet received the attention we believe they deserve. Furthermore, quantitative techno-economical studies of the commercial feasibility and cost versus benefit associated with use cases of cognitive radio crucial in influencing the takeup of the technology by wireless network and service providers but are currently very limited. Our own research is currently focusing on some of the abovementioned technology and business challenges of cognitive access to TVWS.

\section{Acknowledgments}

The author wishes to acknowledge his colleagues at BT, S. Kawade, M. Fitch, X. Gu, K. Briggs, R. MacKenzie, P. Bruce, A. Sago, C. Cheeseman, M. Pine, J. Zhang, N. Edwards, and D. Bryant, for stimulating discussions. He thanks Jonas Kronander from Ericsson $\mathrm{AB}$ for reading the manuscript. The author would like to thank the European Union for providing partial funding of this work through the EU FP7 project INFSO-ICT-248303 QUASAR.

\section{References}

[1] A. Wyglynski, M. Nekovee, and T. Hou, Eds., Cognitive Radio Communication and Networks: Principle and Practice, Academic Press, London, UK, 2010.

[2] J. Mitola III and G. Q. Maguire Jr., "Cognitive radio: making software radios more personal," IEEE Personal Communications, vol. 6, no. 4, pp. 13-18, 1999.

[3] M. Nekovee, "Dynamic spectrum access_concepts and future architectures," BT Technology Journal, vol. 24, no. 2, pp. 111116, 2006.

[4] Federal Communications Commission, "Spectrum policy task force," Report of the Spectrum Efficiency Working Group, November 2004, http://www.fcc.gov/sptf/reports.html/.

[5] Federal Communications Commission (FCC), "Second report and order and memorandum. Opinion and order," Tech. Rep. 08-260, November 2008.

[6] Federal Communications Commission (FCC), "Additional spectrum for unlicensed devices below $900 \mathrm{MHz}$ and in the 3 GHz band," ET Docket 02-380, December 2002.

[7] Federal Communications Commission (FCC), "Unlicensed operation in the TV broadcast bands," ET Docket 04-186, May 2004.

[8] Ofcom, "Digital Dividend Review, A statement on our approach towards awarding the digital dividend," December 2007.

[9] “Ofcom Consultation: Digital Dividend," February 2009, http: //www.ofcom.org.uk/consult/condocs/cognitive/summary/.

[10] Ofcom, "Statement on Cognitive Access to Interleaved Spectrum," July 2009.

[11] J. Bradford, T. Cook, D. Ramsbottom, and S. Jones, "Optimising usage of spectrum below $15 \mathrm{GHz}$ used for defence in the UK," in Proceedings of the IET Seminar on Software Defined Radio and Cognitive Radio, London, UK, September 2008.

[12] M. Nekovee, "Quantifying the availability of TV white spaces for cognitive radio operation in the UK," in Proceedings of the IEEE International Conference on Communications Workshops (ICC '09), Dresden, Germany, June 2009.

[13] S. Kawade and M. Nekovee, "Wireless options for high datarate indoor users: cognitive access to TV white spaces," in Proceedings of the IEEE International Symposium on New Frontiers in Dynamic Spectrum Access Networks (DySPAN '10), Singapore, April 2010.

[14] M. Mishra and A. Sahai, "How much white space is there?" Tech. Rep., Electrical Engineering and Computer Sciences, University of California at Berkeley, January 2009. 
[15] T. X. Brown and D. C. Sicker, "Can cognitive radio support broadband wireless access?" in Proceedings of the 2nd IEEE International Symposium on New Frontiers in Dynamic Spectrum Access Networks (DySPAN '07), pp. 123-132, April 2007.

[16] “CogNeA," http://www.cognea.com/.

[17] K. Challapali, "Philips Research North America (private communication)," FAQ on CogNeA Alliance, K. Kimyacioglu, Philips.

[18] Ecma 392, Ecma International Standard.

[19] J. Wang, M. Sun Song, S. Santhiveeran, et al., "First cognitive PHY/MAC standard for personal/portable devices in TV White Spaces".

[20] “IEEE 802.22," http://www.ieee802.org/22/.

[21] C. Cordeiro, D. Cavalcanti, and S. ShankarA. Wyglynski, M. Nekovee, and T. Hou, "Cognitive radio for broadband wireless access in TV bands: the IEEE 802.22 standards," in Cognitive Radio Communication and Networking: Principle and Practice, Academic Press, Boston, Mass, USA, 2009.

[22] A. Sahai and D. Cabric, "Spectrum sensing: fundamental limits and practical challenges," in Proceedings of the IEEE International Symposium on New Frontiers in Dynamic Spectrum Access Networks (DySPAN '05), Baltimore, Md, USA, November 2005.

[23] T. Yücek and H. Arslan, "A survey of spectrum sensing algorithms for cognitive radio applications," IEEE Communications Surveys and Tutorials, vol. 11, no. 1, pp. 116-130, 2009.

[24] H.-S. Chen, W. Gao, and D. G. Daut, "Spectrum sensing for wireless microphone signals," in Proceedings of the 5th Annual IEEE Communications Society Conference on Sensor, Mesh and Ad Hoc Communications and Networks Workshops, San Francisco, Calif, USA, June 2008.

[25] A. Mossa and A. Jeoti, "Cyclostationary-based spectrum sensing for analog TV and wireless microphone signals," in Proceedings of the 1st International Conference on Computational Intelligence, Communication Systems and Networks, pp. 380-385, 2008.

[26] P. D. Sutton, K. E. Nolan, and L. E. Doyle, "Cyclostationary signatures in practical cognitive radio applications," IEEE Journal on Selected Areas in Communications, vol. 26, no. 1, pp. 13-24, 2008.

[27] L. P. Goh, Z. Lei, and F. Chini, "DVB detector for cognitive radio," in Proceedings of the IEEE International Conference on Communications (ICC'07), pp. 6460-6465, Glasgow, UK, June 2007.

[28] G. Noh, J. Lee, H. Wang, S. You, and D. Hong, "A new spectrum sensing scheme using cyclic prefix for OFDM-based cognitive radio systems," in Proceedings of the IEEE Vehicular Technology Conference (VTC '08), pp. 1891-1895, May 2008.

[29] "BT Response to Ofcom Consultation on Digital Dividend," Cognitive Access.

[30] A. Ghasemi and E. S. Sousa, "Collaborative spectrum sensing for opportunistic access in fading environments," in Proceedings of the 1st IEEE International Symposium on New Frontiers in Dynamic Spectrum Access Networks (DySPAN '05), pp. 131136, Baltimore, Md, USA, November 2005.

[31] G. Ganesan and Y. Li, "Cooperative spectrum sensing in cognitive radio, part I: two user networks," IEEE Transactions on Wireless Communications, vol. 6, no. 6, pp. 2204-2212, 2007.

[32] Y. Selén and J. Kronander, "Cooperative detection of Programme making special event devices in realistic fading environements," in Proceedings of the IEEE International Symposium on New Frontiers in Dynamic Spectrum Access Networks (DySPAN '10), Singapore, April 2010.

[33] R. Rajbanshi, A. M. Wyglinski, and G. J. Minden, "An efficient implementation of NC-OFDM transceivers for cognitive radios," in Proceedings of the 1st International Conference on Cognitive Radio Oriented Wireless Networks and Communications (CROWNCOM '06), June 2006.

[34] R. Rajbanshi, OFDMbased cognitive radio for DSA networks, Ph.D. thesis, University of Kansas, Lawrence, Kan, USA, 2007.

[35] W. Rhee, J. C. Chuang, and L. J. Cimini, "Performance comparison of OFDM and multitone with polyphase filter bank for wireless communications," in Proceedings of the 48th IEEE Vehicular Technology Conference (VTC '98), pp. 768-772, May 1998.

[36] M. Jankiraman, Space-Time Codes and MIMO Systems, Artech House, Boston, Mass, USA, 2004.

[37] N. Devroye, P. Mitran, and V. Tarokh, "Limits on communications in a cognitive radio channel," IEEE Communications Magazine, vol. 44, no. 6, pp. 44-49, 2006.

[38] N. S. Shankar and C. Cordeiro, "Analysis of aggregated interference at DTV receivers in TV bands," in Proceedings of the 3rd International Conference on Cognitive Radio Oriented Wireless Networks and Communications (CROWNCOM '08), Singapore, May 2008.

[39] “UK FREE.TV," http://www.ukfree.tv/.

[40] K. Briggs, “BT innovate and design,” Tech. Rep., July 2009.

[41] P. L. Rice, A. G. Longley, K. A. Norton, and A. P. Barsis, "Transmission loss predictors for tropospheric communication circuits," Tech. Note 101, U S Government Printing Office, Washington, DC, USA, 1965.

[42] E. Obregon and J. Zander, "Short range white space utilization in broadcast systems for indoor environments," in Proceedings of the IEEE International Symposium on New Frontiers in Dynamic Spectrum Access Networks (DySPAN '10), Singapore, April 2010.

[43] M. Nekovee, "Cognitive access to TV White Spaces: spectrum opportunities, commercial applications and remaining technology challanges," in Proceedings of the IEEE International Symposium on New Frontiers in Dynamic Spectrum Access Networks (DySPAN '10), Singapore, April 2010.

[44] D. López-Pérez, A. Valcarce, G. De La Roche, and J. Zhang, "OFDMA femtocells: a roadmap on interference avoidance," IEEE Communications Magazine, vol. 47, no. 9, pp. 41-48, 2009. 

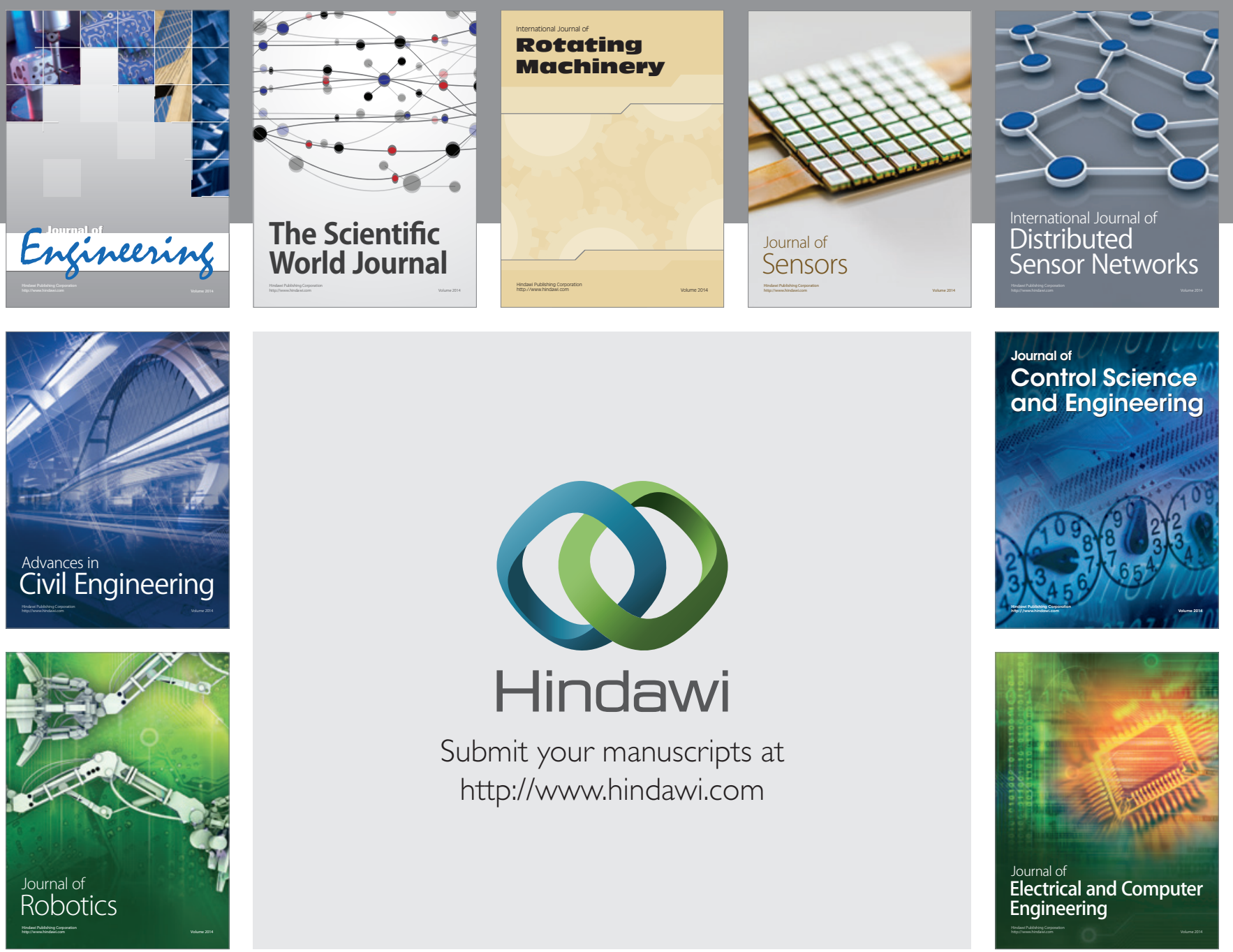

Submit your manuscripts at

http://www.hindawi.com
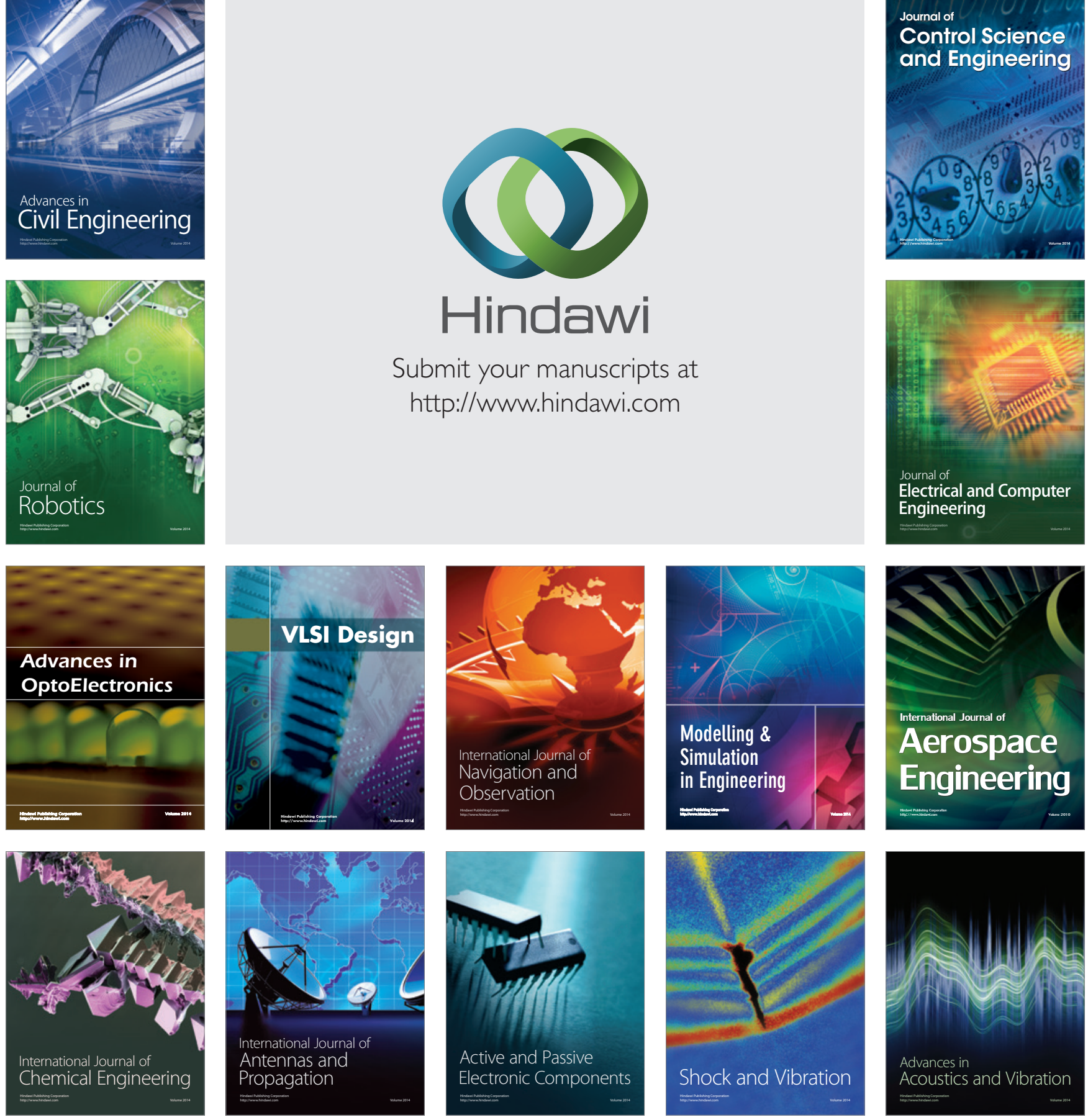